\title{
Le déclin de la technologie générale : Léon Lalanne et l'ascendance de la science des machines
}

The decline of general technology: Léon Lalanne and the ascendancy of applied mechanics

Joost Mertens

\section{OpenEdition}

Édition électronique

URL : https://journals.openedition.org/dht/1749

DOI : $10.4000 /$ dht. 1749

ISSN : 1775-4194

Éditeur :

Centre d'histoire des techniques et de l'environnement du Cnam (CDHTE-Cnam), Société des élèves du CDHTE-Cnam

Édition imprimée

Date de publication : 1 décembre 2011

Pagination : 107-117

ISBN : 978-2-9530779-7-1

ISSN : 0417-8726

\section{Référence électronique}

Joost Mertens, « Le déclin de la technologie générale : Léon Lalanne et l'ascendance de la science des machines », Documents pour l'histoire des techniques [En ligne], 20 | 2e semestre 2011, mis en ligne le 17 septembre 2012, consulté le 21 septembre 2021. URL : http://journals.openedition.org/dht/1749 ; DOI : https://doi.org/10.4000/dht.1749 


\title{
Le déclinde la technologie générale: Léon Lalanne et l'ascendance de la science des machines
}

\section{Joost Mertens}

\begin{abstract}
RéSUMÉ
Dans ses deux écrits sur la technologie (1840, 1843), Léon Lalanne a fait la transition entre le discours technologique classique, centré sur la production artisanale et ses opérations manuelles, et la technologie comme science intermédiaire, centrée sur les machines et leurs explications mécaniques. Dans son Essai philosophique, il est encore tout près des technologues comme Gérard-Joseph Christian, Andrew Ure et André-Marie Ampère, mais la mécanique appliquée figure déjà explicitement dans sa philosophie. Quelques années plus tard, la technologie classique est réduite à une collection de faits divers. La nouvelle technologie se situe dans les domaines de la mécanique, de la cinématique, des forces, des machines, du travail mécanique, des moteurs inanimés. Et les sciences qui s'en occupent sont des sciences intermédiaires, selon les mots de Charles Laboulaye et d'Hélène Vérin. La technologie n'est plus la théorie d'une pratique, mais se situe entre les pratiques et les sciences physiques.
\end{abstract}

Résumés et mots clés en anglais sont regroupés en fin de volume, accompagnés des mots clés français

Q uand, en 1966, Jacques Guillerme et Jan Sebestik écrivent leur étude, par la suite devenue très célèbre, des commencements de la technologie?, ils font également quelques remarques sur sa fin, vers le milieu du XIXe siècle. Cela ressemblait à une lente explosion, et ce qui en restait, comme une espèce de résidu, c'était une collection de sciences appliquées:

" Sans qu'il y ait une coupure nette dans notre exposé, nous nous sommes arrêtés vers le milieu du XIXe siècle. Mais cette limite approximative correspond à la fois à une évanescence et à une dispersion du discours technologique. La multiplication saturante de ses produits provoque son éclatement en savoirs techniques spécialisés et parcellaires. La technologie, c'estalorsles aventures, dans l'industrie, de la science appliquée $\|^{2}$.

1 Jacques Guillerme et Jan Sebestik, « Les commencements de la technologie ॥, Thalès, t. 12, année 1966, Paris, PUF, 1968, pp. 1-72 ; reprint Documents pour l'histoire des techniques, 14, 2007, pp. 50-121.

2 lbid., p. 51 (reprint).
Plusieurs autres auteurs ayant étudié I'histoire du discours technologique ont fait des remarques analogues concernant sa fin. Selon Günter Ropohl, dans sa première esquisse de la technologie beckmannienne revivifiée, ce fut après 1850 que la technologie générale tomba dans l'oubli, par l'établissement notamment des sciences appliquées parcellaires (spezialisierte Technikwissenschaften) ${ }^{3}$. François Sigaut a aussi observé qu'à partir des années 1850, la tradition technologique s'essouffle, après un siècle environ d'efficacité multiple et que l'expression même de technologie entre en désuétude. La technologie est remplacée par "l'idéologie triomphante de la science appliquée ". $^{4}$. Tout récemment, dans un coup d'œil rétrospectif

3 Günter Ropohl, « Prolegomena zu einem neuen Entwurf der allgemeinen Technologie ॥, dans Hans Lenk et Simon Moser éd., Techne Technik Technologie, Pullach, Verlag Dokumentation, 1973, pp. 152-172.

4 François Sigaut, "Haudricourt et la technologie ", dans André-Georges Haudricourt, La technologie, science humaine. Recherches d'histoire et d'ethnologie des techniques, Paris, Maison des sciences de l'homme, 1987, pp. 9-34. 
de Jan Sebestik sur les commencements des études historiques des commencements de la technologie, entreprises par Georges Canquilhem, Jacques Guillerme, Edouard Morère et lui-même, on trouve la remarque suivante : les traités célèbres de Charles Babbage (1833) et d'Andrew Ure (1835) signalaient l'achèvement de la période classique de la technologie générale qui, ensuite, se dispersa, s'étiola, disparut pour être remplacée par les sciences appliquées, " et cette conception a fini par devenir prédominante $\|^{5}$.

Cette mutation de la technologie en science appliquée n'avait rien d'autonome ou d'imprévu. Elle était suscitéé par des développements englobants au niveau de l'économie et de la politique. Guillerme et Sebestik ont signalé l'évolution précipitée et la diversification de l'industrie à partir des années cinquante du XIXe siècle, ainsi que l'établissement du régime libéral. Par là, la technologie est devenue obsolète doublement. La technologie classique avait toujours été liée à l'ancien univers artisanal, mais la production artisanale était en train de mourir. Deuxièmement, l'ancienne technologie sentait le caméralisme, c'est-à-dire l'État, le dirigisme économique et ce n'était pas très libéral. " La technologie », selon Guillerme et Sebestik, " est une discipline contemporaine de la transformation de la société artisanale et manufacturière en société industrielle moderne $11^{6}$, et celle-ci était gagnante. François Sigaut a mis en lumière une raison de plus pour que la technologie soit devenue obsolète. C'est que l'invention et l'innovation techniques ne dépendaient plus des descriptions systématiques des arts et manufactures mais qu'elles étaient inspirées, de plus en plus, par les sciences physiques et chimiques : « À quoi bon désormais ces milliers de volumes de descriptions de techniques obsolètes, ou vouées à le devenir bientôt ? [...] C'est désormais des sciences, et des sciences seules, que l'époque attend les progrès de son industrie 17 .

Léon Lalanne (1811-1892), polytechnicien, ingénieur du corps des ponts et chaussées, inventeur d'un arithmoplanimètre et rédacteur de diverses tables de calcul, écrivit deux essais sur la technologie au début des années 1840. Lalanne doit être considéré comme une figure de transition dont les écrits se situent entre la technologie traditionnelle

5 Jan Sebestik, "Les commencements de la technologie Postface/préface "I, Documents pour I'histoire des techniques, 14, 2007, pp. 123-133.

6 Jacques Guillerme et Jan Sebestik, "Les commencements 》, op. cit. note 1, p. 119 (reprint).

7 François Sigaut, « Haudricourt », op. cit. note 4, pp. 23-24.

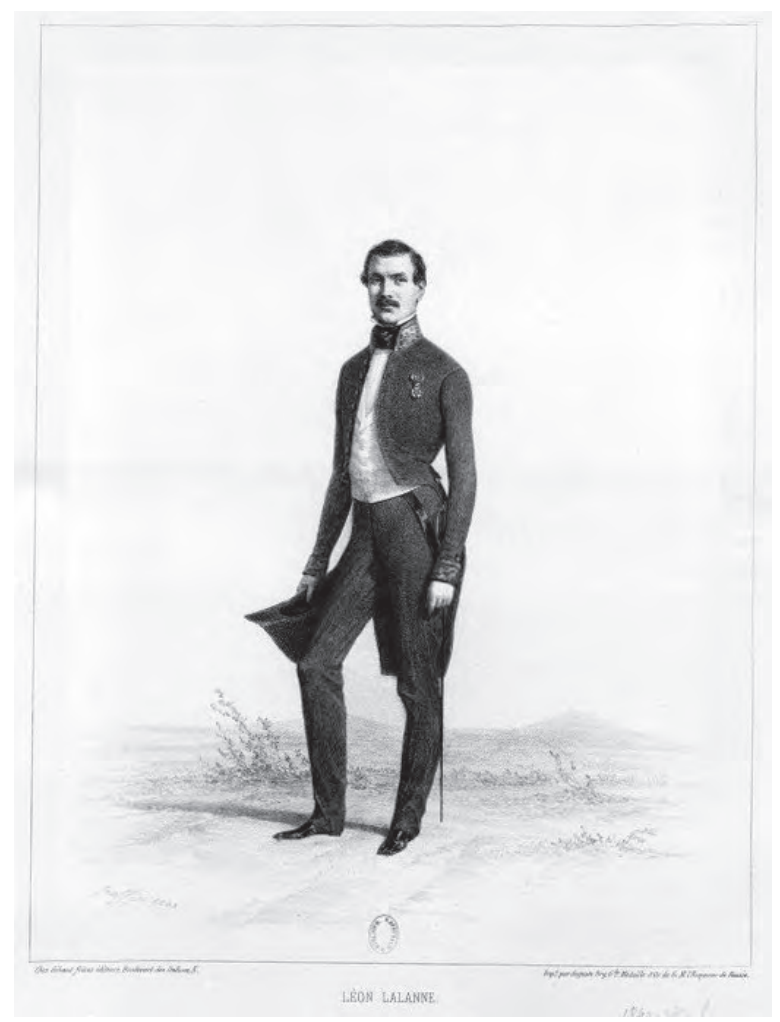

fig. 1. Léon Lalanne (1848) : lithographie d'Auguste Raffe† (Portraits des membres de l'expédition scientifique dans la Russie méridionale en 1837).

et les nouvelles sciences appliquées, surtout la mécanique appliquée. Son premier essai a été publié dans le tome VIII de l'Encyclopédie nouvelle, ensuite comme un opuscule de cinquante-six pages ${ }^{8}$ et réimprimé, en 1850 dans l'Encyclopédie théologique de l'abbé Jacques-Paul Migne'. Son deuxième essai date de 1843 et se trouve dans un aide-mémoire intitulé Un million de faits, maintes fois réédité ${ }^{10}$. Son Essai philosophique de 1840 n'est pas tombé dans I'oubli. Jacques Guillerme, Jan Sebestik et Édouard Morère "l l'ont inclus dans leurs histoires de la technologie. Mais sa contribution « Technologie » au

8 Léon Lalanne, Essai philosophique sur la technologie, Bourgogne et Martinet, 1840.

9 Anon. [Léon Lalanne], «Technologie », dans Jacques-Paul Migne éd., Encyclopédie théologique, t. 42, Dictionnaire d'astronomie, de physique et de météorologie, 1850, pp. 1218-1252.

10 Léon Lalanne, "Technologie ", dans Jean Aicard, Léon Lalanne e.a., Un million de faits : Aide-mémoire universel des sciences, des arts, et des lettres, $4^{4}$ édn., Bruxelles, Société typographique belge, 1846, pp. 203-226.

11 Édouard Morère, "Les vicissitudes du sens de "technologie" au début du XIXe siècle 1 , Thalès, t. 12, année 1966, Paris, PUF, 1968, pp. 73-84. 
Million de faits n'est pas souvent étudiée, alors qu'elle révèle très clairement comment Lalanne bascule de la technologie classique vers la science des machines. Une lecture de ces deux ouvrages peut mettre en lumière, d'une façon un peu détaillée, comment les sciences appliquées ont triomphé de la technologie.

\section{Essai philosophique sur la technologie}

En guise de préliminaires, voici quelques remarques sur l'emploi un peu nonchalant des termes " technologie " et " technologique " par Lalanne. Cette nonchalance est peut-être déjà le signe du déclin de la technologie générale et de l'indétermination concomitante du sens de ces expressions. Morère l'a signalé : «Constamment utilisé par Lalanne, le terme technologie signifie technique, ensemble de procédés utilisés à moment donné $11^{12}$. Cet usage anglo-saxon se dessine clairement quand Lalanne parle de procédés technologiques, qui ne sont pas des manières de décrire la technique mais simplement des procédés techniques, des suites d'opérations industrielles. Aussi, quand Lalanne parle de l'histoire de la technologie, il ne se réfère pas à I'histoire de la tradition technologique de Beckmann à Ure mais simplement à l'histoire des techniques. Néanmoins, il est aussi conscient du sens original du terme. Ainsi, la technologie est « le corps de doctrine embrassant tous les procédés matériels des arts $\|^{13}$ et la technologie spéciale ou descriptive embrasse «les descriptions particulières des procédés industriels ", par exemple celles des vingt-deux volumes du Dictionnaire technologique de Lenormand et Francœur ${ }^{14}$.

Definition, classification, énumération des arts industriels "La connaissance de tous les procédés des arts", c'est la définition quasi sténographique de la technologie selon Lalanne. Mais aussitôt après l'avoir formulée, il introduit une limite. La technologie proprement dite exclut l'agriculture, l'art de produire des végétaux ainsi que la zootechnie, l'art de dominer, d'asservir et d'utiliser les animaux. En ceci, Lalanne ne s'écarte point des analyses qui étaient d'usage dans la technologie générale. Christian, et, plus tard, Ure aussi, introduisent la même exclusion de l'agriculture et de l'élevage ${ }^{15}$. Selon Christian, le

\section{Ibid., p. 82.}

13 Lalanne, «Technologie », op. cit. note 10, p. 226.

$14 \mathrm{lbid}$.

15 Joost Mertens, "The mere handicrafts : Ure's Dictionary (1839-1853) compared with the Dictionnaire technologique (1822-1835) ", Irina Gouzévitch et Liliane travail industriel comporte trois séries : se procurer des matières premières (l'agriculture) ; opérer leur transformation industrielle; faire circuler les produits issus de cette transformation (le commerce). Mais la seconde série, celle de "la transformation des matières premières et la préparation de diverses combinaisons inorganiques " est ce qui s'appelle le travail industriel proprement dit' ${ }^{16}$. En 1839, Andrew Ure proposait une structure tripartite pour l'industrie manufacturière, fort analogue à celle de Christian : la production « primaire » des matières premières par l'agriculture, l'élevage, la pêche et l'industrie minière, ensuite la transformation mécanique ou chimique de ces matières premières, et finalement la distribution des produits finis. Mais, de la même manière, le sujet d'étude du technologue doit étre l'industrie manufacturière proprement dite, c'est-à-dire "la transformation des matières premières, par l'action mécanique ou chimique, en des objets d'une valeur d'échange $11^{17}$.

L'argument en faveur de cette exclusion semble se réduire à une sorte d'hésitation devant l'extension du discours technologique à la manipulation des êtres vivants. Pour Christian, le principe de l'agriculture, " c'est la nature, c'est une force productrice que I'homme ne peut ni expliquer ni commander $"$. L'agriculture se distingue de la chimie «par l'action nécessaire d'une force, celle de la végétation, qui échappe entièrement aux principes de la science ; action inexplicable qui met l'agriculture dans une catégorie toute particulière $11^{18}$. Pour Ure, les matières premières de l'industrie manufacturière proprement dite proviennent du progrès organique des végétaux et des animaux qui se déroule avec ou sans les soins attentifs (the fostering care) de l'homme ${ }^{19}$. Et manifestement, les soins attentifs ne s'identifient pas aux opérations techniques. Pour Lalanne, il est vrai que la culture de la terre et la conservation ou la capture des animaux sont exercées en employant beaucoup d'outils, d'instruments, de machines, de constructions et de procédés techniques. Toutefois, «l'intervention de la force vitale propre aux êtres

Pérez éd., " Les échanges techniques entre la France et l'Angleterre (XVIe-XIXe siècles). RéseauX, comparaisons, représentations 11, Documents pour l'histoire des techniques, $\mathrm{n}^{\circ} 18,2009$, pp. 277-285.

16 Gérard-Joseph Christian, Vues sur le système général des opérations industrielles, ou Plan de technonomie, Paris, Mme Huzard et Mme Courcier, 1819, p. 43.

17 Andrew Ure, Dictionary of arts, manufactures, and mines, Londres, Longman, etc., 1839, p. iii.

18 G.-J. Christian, op. cit. note 16, pp. 41-42.

19 A. Ure, op. cit. note 17, p. iii. 


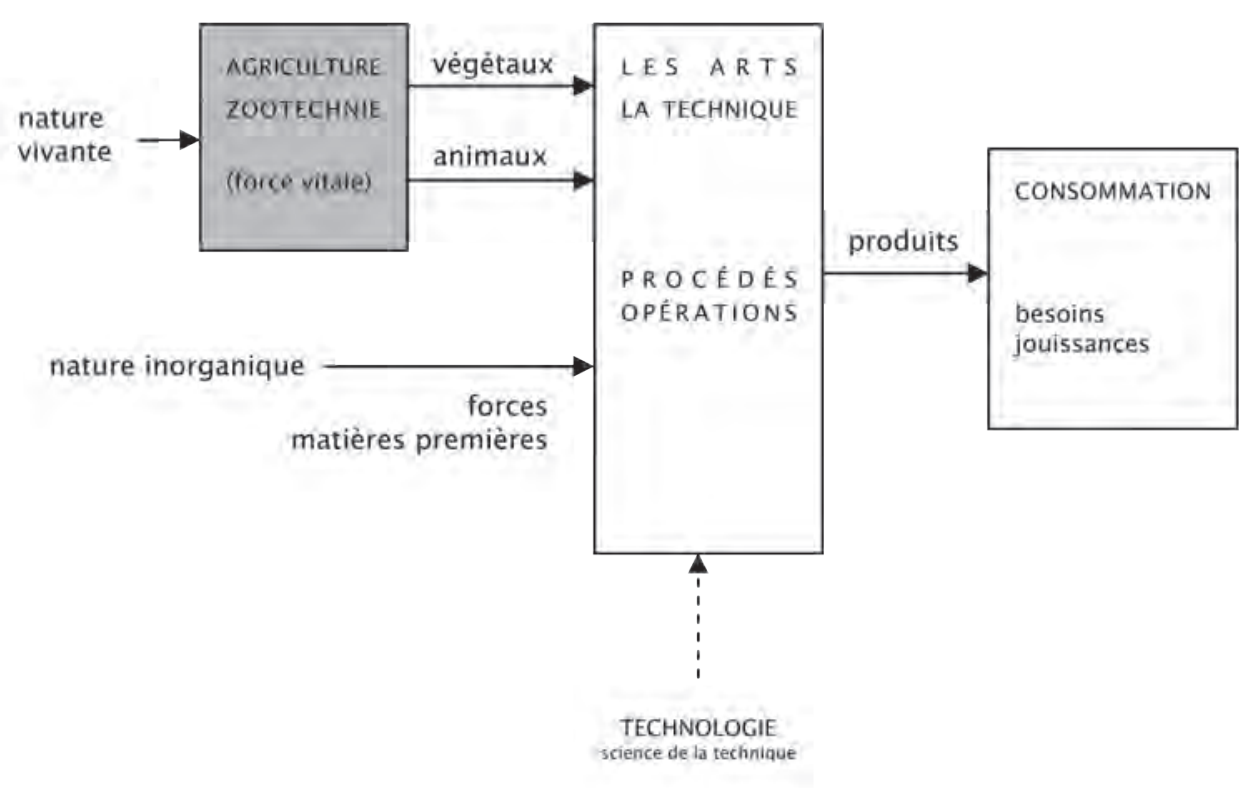

compris dans le règne végétal et dans le règne animal, établit une ligne de démarcation tranchée $12^{20}$ entre la technique proprement dite d'une part et les pratiques de l'agriculture et de la zootechnie d'autre part.

Donc, la technologie dans ce sens restreint est définie comme :

" La science des procédés par lesquels l'homme agit sur les forces et sur les matières premières fournies par la nature organique et inorganique, pour approprier ces forces et ces matières à ses besoins et à ses jouissances. $11^{21}$

Le schéma ci-dessus montre la structure générale de la technologie "à la Lalanne ». Les pratiques de l'agriculture et de l'élevage produisent des forces et des matières premières organiques, par exemple les huiles pour le savonnier, les "forces" des bêtes de somme et des animaux de trait, le noir animal pour l'industrie sucrière.

Quant à la classification des arts industriels, avant de présenter ses sept classes de transformations techniques, Lalanne critique les catégories mises en avant par l'Encyclopédie, l'ordre alphabétique de Lenormand et les simplifications d'Andrew Ure.

Diderot et d'Alembert avaient rangé les arts, métiers et manufactures selon leur input, la matière première travaillée, la substance à façonner. Selon Lalanne, cette optique a mené à la décision absurde de placer le vitrier et l'opticien dans la méme

20 L. Lalanne, op. cit. note 8, p. 7. $21 \mathrm{lbid}$. catégorie, celle du verre, alors que ces métiers ne montrent que peu d'analogies entre eux. D'autre part, des métiers fort analogues, comme ceux du tailleur et du gantier, se trouvent dans des classes éloignées.

Lalanne a cherché, en vain, un tableau méthodique des arts dans le Dictionnaire technologique de Lenormand et Francœur « quoiqu'il ait été annoncé à la fin de l'introduction $\|^{22}$. II est vrai que les éditeurs de ce dictionnaire ont promis de terminer leur ouvrage par une " classification générale et méthodique de tous les Arts », et qu'ils ne se sont pas acquittés de cette promesse. Mais il est vrai aussi, et Lalanne ne le relève pas, que Lenormand a défendu explicitement l'absence de toute classification «plus ou moins contestable » et son remplacement par l'ordre alphabétique augmenté avec des renvois aux arts analogues ou contigus ${ }^{23}$.

La classification des arts mécaniques selon Andrew Ure n'était pas fondée comme celle de l'Encyclopédie sur la nature des substances travaillées, mais sur treize propriétés fondamentales, comme la divisibilité, la perméabilité, l'élasticité, etc. Comme disait Ure, «le vrai principe philosophique pour classer les manufactures mécaniques, est de les disposer selon l'ordre des propriétés générales de la matière [...] sur lesquelles on fait agir les forces mécaniques, à l'effet de changer la configuration des corps pour

22 Ibid., p. 10.

23 Louis-Sébastien Lenormand, "Discours préliminaire ", Dictionnaire technologique, t. 1, 1822, pp. xxxvii-xli. Voir aussi Joost Mertens, «Technology as the science of the industrial arts : Louis-Sébastien Lenormand (1757-1837) and the popularization of technology ", History and Technology, 18, 2002, pp. 203-231.

110 Documents pour l'histoire des techniques $\mathrm{n}^{\circ} 20$ - décembre 2011 


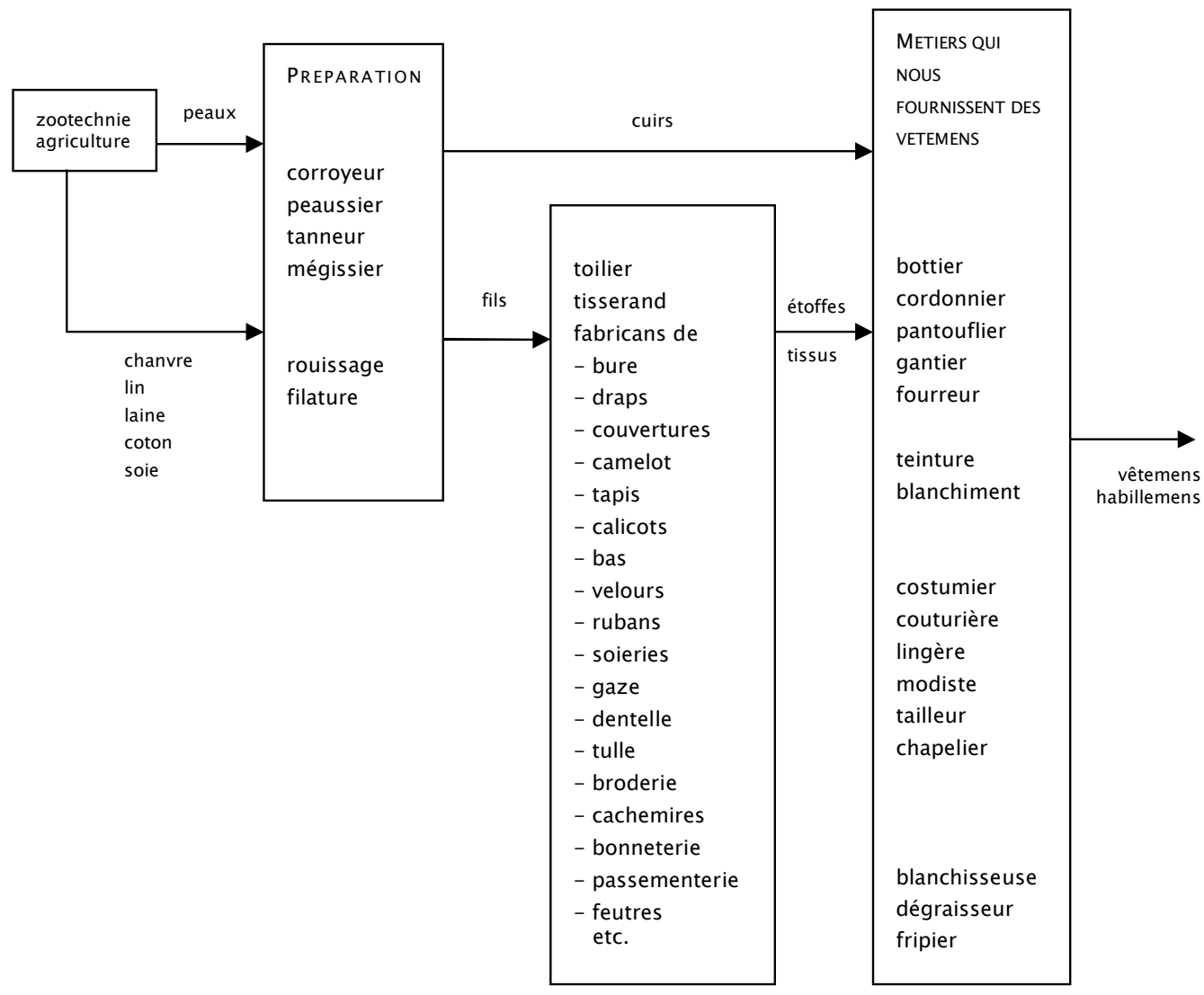

les adapter aux usages de la vie $12^{24}$. Selon Lalanne, la classification d'Ure présentait " le vice radical » de négliger entièrement le fait que tout procédé technique est une concaténation d'actes opératoires singuliers. ॥ n'y a pas de procédés techniques qui agissent sur une propriété unique de la matière : « II serait difficile de citer beaucoup de procédés, où la plupart des propriétés physiques essentielles à la matière ne soient pas mises en jeu successivement ou à la fois $12^{25}$. C'està-dire : un procédé technique est (presque) toujours un enchaînement en série d'opérations discrètes (ou " monades opératoires ») et bien souvent un enchaînement en parallèle de telles séries.

Pour pallier ces défauts, radicaux ou non, Lalanne élabore une classification fondée sur une série de besoins, fondamentaux ou dérivés. Les sept classes qu'il distingue ont pour but de satisfaire divers besoins, tels la subsistance, la santé, l'habit, le logement, le transport, les soins du corps, l'instruction. Cette classification, plus ou moins contestable, pour reprendre le mot de Lenormand, est analogue à celle établie, en 1834, par Charles Dupin, à l'occasion

24 Andrew Ure, Philosophie des manufactures, Paris, Mathias, 1836, pp. 87-88.

25 L. Lalanne, op. cit. note 8, p. 10. de la huitième exposition nationale des produits de l'industrie française ${ }^{26}$. Le critère de classification que Dupin proposait était le but social d'un art industriel, le genre de services rendus à l'homme.

À titre d'exemple, voici la catégorie des vêtements. Elle peutmontrerla position des techniques improprement dites, l'idée de la concaténation (entre végétaux/peaux et habillement), et la position de la première catégorie, celle de la préparation des matières premières.

On voit que Lalanne n'exclut pas, comme le fait Ure, les métiers traditionnels. Figurent aussi, sur les pages de son Essai philosophique, les arts du boulanger, du vinaigrier, du limonadier, du charpentier, du ferblantier, du quincailler, etc. L'Essai de Lalanne se situe donc pleinement dans la technologie classique. Mais un changement intervient dans les paragraphes suivants, sur les sciences appliquées et la nature des procédés techniques.

Les sciences appliquées

Il est d'usage entre technologues, dit Lalanne, de distinguer les arts chimiques et physiques des arts mécaniques et de calcul. Mais cette distinction, selon

26 Charles Dupin, Rapport du jury central sur les les produits de l'industrie française exposés en 1834, T. I, Paris, Imprimerie royale, 1836, pp. 4-5. 
lui, ne s'applique point aux procédés mis en œuvre dans les industries réelles. L'exemple de la production du sucre indigène le prouve. La transformation des betteraves en sirop, et ensuite du sirop en sucre, comporte cinq opérations physico-chimiques : la dessiccation, la formation, la concentration, la clarification, la cristallisation. Mais toutes ces opérations sont effectuées par des moyens mécaniques. L'industrie sucrière est pleine de machines à agiter, tamiser, pomper, presser, verser, mélanger les substances et les dissolutions à travailler. E† cette partie de l'industrie sucrière fait l'objet de la science des machines : " La disposition du moteur et des organes destinés à transmettre les forces nécessaires à la mise en œuvre des actions chimiques, font partie de la science des machines $11^{27}$.

Ceci est vrai non seulement pour l'industrie sucrière mais pour presque toutes les industries modernes, qu'elles soient physiques ou chimiques. En 1840, selon Lalanne, la production industrielle était donc entièrement mécanisée ou presque.

Deux conclusions peuvent en être tirées. Premièrement, Lalanne commence à négliger la production artisanale et les arts traditionnels. Deuxièmement, parmi les sciences appliquées, c'est la mécanique appliquée qui reçoit une certaine préférence. La science des machines se trouve au cœur de la technologie pour ainsi dire.

Il y a une raison de plus pour la préférence donnée à la mécanique appliquée. Le tableau des sciences physiques et chimiques appliquées n'était pas dessiné très clairement tandis que la science des machines se composait d'un nombre restreint de lois fondamentales: "Nous ne pouvons pas entreprendre ici de donner même une simple esquisse des procédés de la technologie chimique et physique, dont la nature et les moyens sont variés à l'infini, et ne peuvent être formulés en quelques lois simples comme ceux de la mécanique appliquée. $12^{28}$

Peut-être qu'en 1840, la physique et la chimie appliquées n'en étaient encore qu'à leurs balbutiements ? Peut-être étaient-elles encore à développer ? Lalanne, en tout cas, préfère la précision de la mécanique appliquée à la collection disparate et imprécise des technologies physiques et chimiques.

Une partie importante de la mécanique appliquée consiste en l'analyse des "forces " employées dans la production mécanisée. Lalanne propose ainsi de distinguer les arts chimiques et

27 L. Lalanne, op. cit. note 8, pp. 16-17.

28 lbid., p. 23. physiques des arts mécaniques et de calcul selon : (a) la force musculaire de l'homme et des animaux, celle des moteurs animés, (b) les agens naturels, l'eau et le vent, employés par exemple dans les moulins à vent hollandais et les roves hydrauliques françaises, (c) surtout la vapeur, appliquée dans les mines, l'industrie manufacturière, les locomotives, les bateaux à vapeur. Les moteurs animés étaient en train de disparaître du paysage industriel :

" La tendance évidente de la technologie [le développement technique, $\mathrm{NdA}$ ] est de substituer de plus en plus la force de la matière brute à celle des animaux, et de consacrer exclusivement le travail de l'homme aux pratiques qui exigent de l'attention $11^{29}$.

Ce travail « attentif » est le même travail que celui qu'Andrew Ure avait encore toléré chez ses « vastes automates $»$ producteurs :

« Dans ces vastes ateliers, le pouvoir bienfaisant de la vapeur appelle autour de lui ses myriades de sujets, et assigne à chacun sa tâche obligée, substituant à leurs pénibles efforts musculaires l'énergie de son bras gigantesque, et ne leur demandant en récompense que leur attention et leur adresse pour rectifier les erreurs légères qui se glissent parfois dans son ouvrage [...] Le but constant et la tendance de tout perfectionnement dans le mécanisme est en effet de se passer entièrement du travail de l'homme, ou d'en diminuer le prix, en substituant l'industrie des femmes et des enfans à celle de l'ouvrier adulte; ou le travail d'ouvriers grossiers à celui de l'habile artisan $1^{30}$.

On voit qu'en détournant la technologie vers la science des machines, Lalanne n'a plus d'yeux pour le travail artisanal, le maniement d'outils, les gestes et les actes opératoires compliqués de ce type de travail, l'habileté, l'adresse et la dextérité des opérations manuelles. En 1819, Christian avait encore présenté une analyse fondamentale du travail industriel « à la mode du métier $\|{ }^{31}$ Pour Lalanne, le travail humain se réduit à la force exercée par un moteur animé et à l'attention d'un ouvrier auprès d'une machine. E† l'énumération de tant de métiers sur les premières pages de son Essai ne paraît plus être qu'un rituel.

29 Ibid., p. 17.

30 A. Ure, op. cit. note 24, pp. 26-35.

31 G.-J. Christian, op. cit. note 16, pp. 44-55.

112 Documents pour l'histoire des techniques n 20 - décembre 2011 


\section{TECHNOLOGIE}

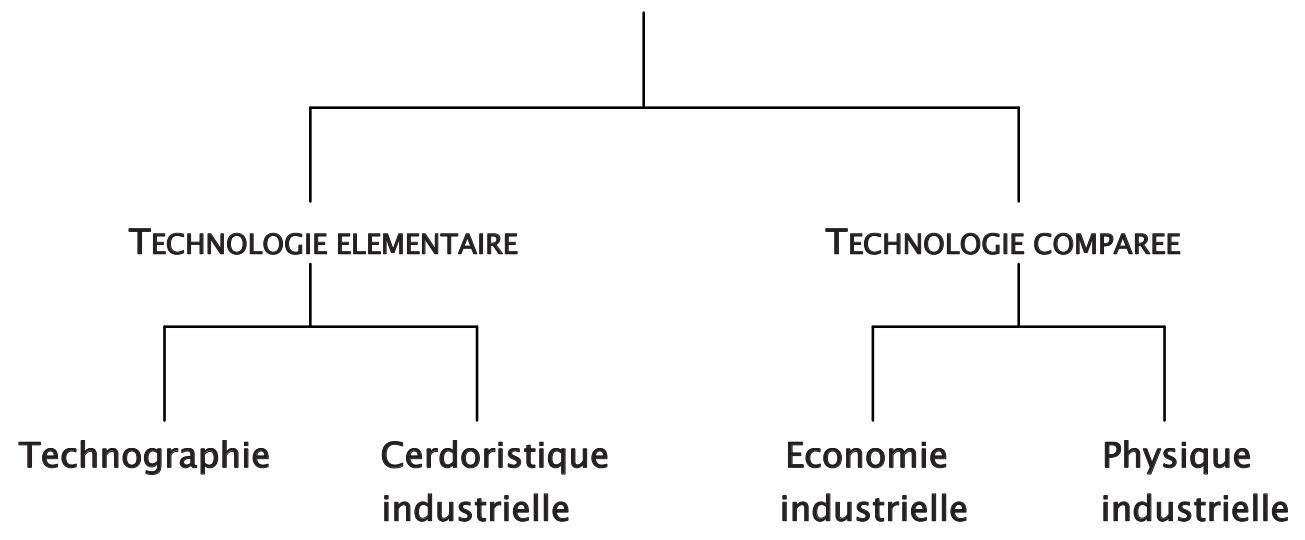

La cerdoristique et la division du travail

La cerdoristique, un néologisme datant de 1834, qu'André Ampère a tenté, en vain, d'introduire dans la langue française, est la science qui calcule et prévoit les profits et les pertes à réaliser dans les entreprises industrielles (ou agricoles). Elle fait partie d'une science autonome ${ }^{32}$, la Technologie, dont l'objet central est le procédé technique et dont les théories portent sur "le choix des moyens les plus propres à atteindre le but qu'on se propose $1^{33}$.

Cette Technologie se compose de quatre disciplines. La première, la technographie, embrasse la connaissance des procédés par lesquels on opère les transformations qu'on fait subir aux corps inorganiques en employant des instruments et des machines. ${ }^{34}$ La technographie est donc ce que d'autres technologues avaient appelée la technologie spéciale ou descriptive et ce que Lalanne avait défini comme "la science des procédés techniques 1 .

32 Hélène Vérin a clairement distingué la technologie comme science autonome de la technologie comme science intermédiaire (ou science "appliquée »). En un sens, Lalanne fait la transition entre ces deux types de technologie. La technologie d'Ampére, comme celles de Beckmann et de Christian, est une science autonome, puisqu'elle a pour objet d'étude l'opération technique, l'acte opératoire, le procédé industriel. C'est donc une science sociale, théorie de l'action intentionnelle. Hélène Vérin, « La technologie : science autonome ou science intermédiaire ", Documents pour l'histoire des techniques, 14, 2007, pp. 134-143. Voir aussi Sigaut, op. cit. note 4.

33 André-Marie Ampère, Essai sur la philosophie des sciences, Paris, Bachelier, 1838, t. 1, p. 85.

34 Ibid., p. 80.

La deuxième discipline, la cerdoristique ou compte de profits et pertes est l'examen des dépenses pour les locaux de production, les appareils nécessaires, les matières premières et la main-d'œuvre, ainsi que du rendement après la vente des choses produites. Le noyau dur de la cerdoristique est évidemment l'art de tenir les livres de comptes.

Ces deux premières parties (la " technologie élémentaire " selon les définitions d'Ampère) s'occupent des transformations techniques d'un caractère standardisé ou routinier. Tant que le technologue, en faisant ses analyses descriptives et comptables, se borne aux procédés existants ou usuels, «il n'apprend qu'à répéter ce qu'on fait dans le lieu qu'il habite, il reste sous le joug de la routine $11^{35}$. La technologie comparée, par contre, s'occupe des aspects dynamiques de la technique. Elle introduit, d'une manière explicite, les idées du progrès et de l'innovation dans le discours technologique. « Pour que l'industrie puisse faire des progrès, il est nécessaire de comparer les procédés, les instrumens, les machines, etc., usités en différens temps et en différens lieux $\|^{36}$. L'une des deux branches de cette technologie comparée, l'économie industrielle, est une discipline empirique, puisqu'elle compare entre elles les transformations industrielles analogues, effectuées ailleurs ou par le passé. Les analyses comparées de l'économie industrielle ont mené à la découverte de l'utilité de la division du travail, toujours selon Ampère.

L'autre branche, la physique industrielle ou science appliquée ou encore science intermédiaire,

35 Ibid., p. 81.

$36 \mathrm{lbid}$. 
compare les procédés existants avec des procédés projetés qu'on a imaginés sur la base d'une analyse causale des procédés suivis jusqu' alors, en appliquant les théories physiques et chimiques pertinentes. La physique industrielle est une science prospective, pronostique :

" II est un autre mode de comparaison, qui consiste à s'aider des connaissances de physique générale [y comprise la chimie], acquises précédemment, à étudier les phénomènes qui se passent dans les transformations que les arts font subir aux corps, à remonter à leurs causes, à prévoir ainsi les résultats qu'on peut attendre des nouveaux procédés et des nouvelles machines, avant d'en faire essai $1^{37}$.

Lalanne adopte les raisonnements d'Ampère mais sans les finesses des distinctions ampériennes et en considérant la cerdoristique seulement comme partie intégrante de l'économie industrielle. Celleci, dit-il, est une discipline fort nouvelle qui n'est pas encore sortie de l'enfance. Le seul à avoir écrit sur ce sujet est Charles Babbage. ${ }^{38}$ Lalanne lui emprunte, souvent littéralement, la théorie de la division du travail, y compris la fabrication des épingles selon Adam Smith, les formulations de Melchiorre Gioja datant de 1815 et les tables du cadastre de Gaspard de Prony. Cette division du travail entre les individus qui concourent à la production du produit manufacturé soulève un problème moral : " La cerdoristique, comme elle est obligée de le faire aujourd'hui, se renfermant uniquement dans son objet, qui est la production économique, laisse de côté la personne morale de l'ouvrier, et ne considère celui-ci que comme un producteur et un directeur de force vive $1^{39}$. Ce qui a des conséquences désastreuses pour la dignité de l'homme, qui, par la répétition indéfinie des mêmes opérations mécaniques, développe certains muscles aux dépens de l'organisme général. Mais, comme l'a vu et propagé Andrew Ure, la mécanisation tend à oblitérer toutes ces questions morales. La science des machines n'est peut-être pas une science humaine, elle est sûrement une science humaniste : "Le principe de la division du travail cesse de choquer l'intelligence et la dignité de l'homme, dès qu'il est appliqué à des organes mécaniques.

37 Ibid., p. 82

38 Charles Babbage, Traité sur l'économie des machines et des manufactures, Paris, Bachelier, 1833.

39 L. Lalanne, op. cit. note 8, pp. 43-44.
C'est dans ce sens qu'il est véritablement appelé à prendre une extension indéfinie à mesure que la science des machines se développe davantage $11^{40}$.

On peut donc conclure qu'en 1840 Lalanne est encore un représentant de la technologie classique, témoin sa définition de celle-ci, ses discussions classificatoires et son énumération technographique qui embrasse tous les métiers traditionnels. En même temps, il s'éloigne déjà de cette tradition vers une conception plus étroite de la technologie comme science - intermédiaire - des machines. Sa négligence du travail artisanal en témoigne, ainsi que son insistance sur les moteurs animés et sur les sciences appliquées, notamment la mécanique appliquée.

Quelques années plus tard, cet éloignement sera plus net.

\section{Un million de faits}

Cette nouvelle contribution à la philosophie de la technologie s'ouvre sur une définition plutôt rituelle: " Le mot Technologie [...] désigne l'ensemble des connaissances relatives aux procédés par lesquels l'homme agit sur la matière pour l'approprier à ses besoins ou à ses jouissances $11^{41}$. En 1840, Lalanne avait poursuivi son exposé par une esquisse de sept classes de pratiques industrielles. Dans ce nouvel essai, cette partie est fortement raccourcie, les considérations classificatoires ne s'y trouvent plus, les sept catégories ont été réduites à quatre (la nourriture, les vêtements, le logement, les arts et les sciences) et, fort significativement, ces descriptions technologiques ont été déplacées à la fin de l'article, comme une sorte d'appendice et réduites à des faits divers ${ }^{42}$.

Le corps de l'article traite de la mécanique appliquée. Lalanne précise qu'il a aussi écrit des contributions sur la physique et sur la mécanique ${ }^{43}$, mais seulement du point de vue scientifique. II faut que ces théories soient développées en un sens pratique pour qu'une technologie apparaisse : " La mécanique et la physique n'ont été esquissées que sous le point de vue scientifique, et il est nécessaire de leur donner un développement particulier, parce qu'elles président à la plupart des procédés de la technologie, et parce que nous devons enseigner la manière de calculer et d'employer les forces qui sont de leur domaine ${ }^{44}$.

\section{Ibid., p. 47.}

41 L. Lalanne, op. cit. note 10, p. 203.

42 lbid., pp. 220-226.

43 Léon Lalanne, "Mécanique » ; " Physique », dans Aicard, op. cit. note 10, pp. 73-82; 106-117.

44 L. Lalanne, op. cit. note 10, p. 203. 


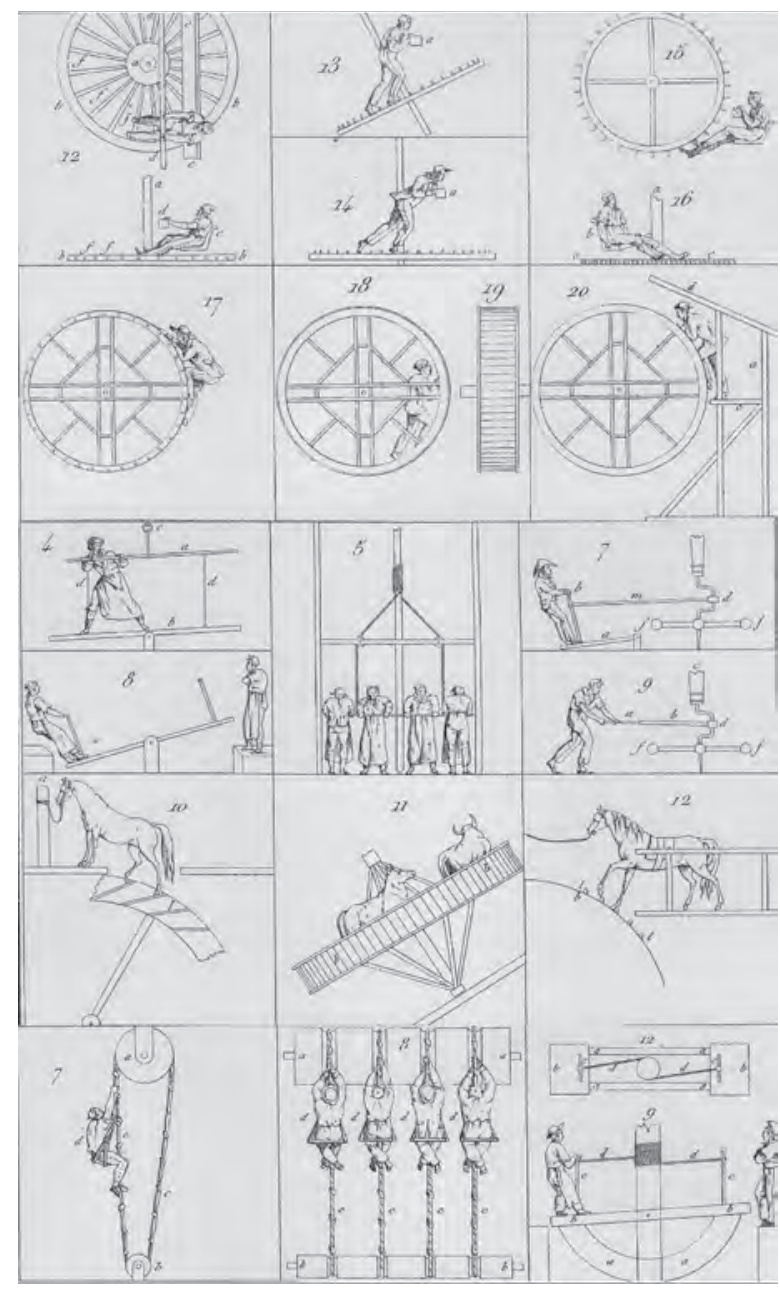

fig. 2. Moteurs animés. Joseph-Antoine Borgnis, Traité complet de mécanique appliquée aux arts, †. 1er, 1818, planches 1,2,3.

La mécanique appliquée se compose de deux parties : la cinématique et la théorie des forces. La cinématique, autre néologisme ampérien, est la mécanique sans les forces. C'est « une science où les mouvements sont envisagés en eux-mêmes, tels que nous les observons dans les corps qui nous environnent, et spécialement dans les machines, indépendamment de la considération des forces qui peuvent produire ces mouvements $\|^{45}$. Elle est "l'introduction à la mécanique industrielle », discipline qui devient la nouvelle définition de la technologie. Celle-ci ne fait plus référence aux actions humaines. Les objets de la mécanique industrielle sont les machines, leur composition et leur fonctionnement.

L'idée maîtresse de la seconde partie de la mécanique industrielle est le travail mécanique. Pour la préciser, Lalanne cite Poncelet : « Travailler, c'est vaincre ou détruire, pour le besoin des arts, des résistances telles que la force d'adhérence des

45 Ibid. molécules des corps, la force des ressorts, celle de pesanteur, l'inertie de la matière, etc. User, polir un corps par le frottement, le diviser en parties, élever des fardeaux, traîner une voiture le long des chemins, bander un ressort, lancer des pierres, des boulets, etc., c'est travailler, c'est vaincre, pendant un certain temps, des résistances sans cesse renouvelées dans la durée de ce temps. $11^{46}$ Lalanne pouvait puiser dans toute une tradition de mécanique appliquée ou mécanique industrielle ou science des machines ${ }^{47}$, qui avait proposé des synonymes divers pour "travail mécanique ": puissance mécanique (Smeaton), moment d'activité (Carnot), effet dynamique (Hachette), quantité d'action (Coulomb, Navier).

E† l'homme, dans tout cela? Son poids et ses muscles figurent parmi les forces qui servent à actionner toutes sortes de machines. L'homme est un moteur animé, tout comme le cheval, le bœuf et le chien. Ainsi, la nouvelle technologie ne s'intéresse plus aux gestes raffinés du travail compliqué des artisans, mais ne s'entend que comme mécanique appliquée de la force musculaire. Et cette force est sur le même plan

46 Ibid., pp. 207-208.

47 Les sources de Lalanne : Lazare Carnot, Essai sur les machines en général (1783) ; Idem, Principes fondamentaux de l'équilibre et du mouvement (1803) ; José Maria Lanz et Agustín de Betancourt, Essai sur la composition des machines (1808) ; John Smeaton, Recherches expérimentales sur l'eau et le vent (1810) ; André Guenyveau, Essai sur la science des machines (1810) ; Jean-Nicolas-Pierre Hachette, Traité élémentaire des machines (1811) ; Joseph-Antoine Borgnis, Traité complet de mécanique appliquée aux arts (1818) ; Charles-Augustin Coulomb, Théorie des machines simples en ayant égard au frottement de leurs parties et la roideur des cordages (1821) ; J.-A. Borgnis, Théorie de la mécanique usuelle, ou Introduction à l'étude de la mécanique appliquée aux arts (1821) ; Idem, Dictionnaire de mécanique appliquée aux arts (1823) ; Gérard-Joseph Christian, Traité de mécanique industrielle (1825) ; Charles Dupin, Géométrie et mécanique des arts et métiers et des beaux-arts (1827) ; Gaspard-Gustave de Coriolis, Calcul de l'effet des machines (1829) ; Claude-Louis Navier, Résumé des leçons données à l'Ecole des ponts et chaussées, sur l'application de la mécanique à l'établissement des constructions et des machines (1833) : Stéphane Flachat, Traité élémentaire de mécanique industrielle (1835) ; A. Taffe, Application des principes de mécanique aux machines (1835) ; G.-G. de Coriolis, Résumés des leçons données à l'École des ponts et chaussées sur l'application de la mécanique à l'établissement des machines (1837) ; Arthur Morin, Aide-mémoire de mécanique pratique (1837) ; Jean-Victor Poncelet, Introduction à la mécanique industrielle, physique ou expérimentale (1839). 
que le vent, l'eau et la vapeur. L'homme, le cheval, le moulin à vent, la rove hydraulique, la machine à vapeur, sont tous des moteurs industriels comparables.

En 1835, selon Stéphane Flachat, la " civilisation» tendait à rendre superflus ces moteurs animés, principalement parce que l'emploi de ceux-ci soulevait un problème moral (ou politique), comme le disait aussi Lalanne :

" Les hommes et les animaux sont des moteurs; leur force, résultat de leur volonté, est un immense moyen mécanique, auquel les progrès de la civilisation tendent à substituer de plus en plus, au moins pour toutes les opérations longues et pénibles, les forces d'autres agens que nous fournit la nature [...] Tandis que, dans les applications des moteurs inanimés, toute amélioration doit être, en définitive, envisagée du point de vue de l'économie, et devient, en un mot, une question d'argent, dans l'emploi des moteurs animés, la question d'humanité est toujours à côté de celle de l'intérêt. II est triste d'être obligé de reconnaître que, dans l'état actuel de l'organisation du travail, la question d'argent est trop souvent encore celle qui passe en première ligne $11^{48}$.

Le moteur favori du technologue moderne, c'est la machine à vapeur. Lalanne consacre moins d'une page aux moteurs animés, environ une page aux roues hydrauliques et aux moulins à vent, mais huit pages à la machine à vapeur. Donc, le cœur de la technologie moderne est la science des machines y compris la cinématique, appliquée surtout à la machine à vapeur. C'est le reflet exact de la transformation de la société artisanale en société industrielle "à vapeur ", indiquée par Jacques Guillerme et Jan Sebestik comme raison principale du déclin de la technologie classique.

À la fin de son article, Lalanne fait quelques remarques sur les écrits technologiques. II apparaît que, quant à la technologie générale, il n'est pas au fait des commencements allemands de la technologie, ni de la technonomie de Christian. II pense que la technologie « considérée comme corps de doctrines embrassant tous les procédés matériels des arts, est née d'hier ") et que l'expression même est récente, peut-être encore un néologisme ampérien. Lalanne connaît les ouvrages de Charles Babbage et

48 Stéphane Flachat, Traité élémentaire de mécanique industrielle, Paris, Tenré, 1835, op. cit. note 47, pp. 41, 53.
d'Andrew Ure mais il ne les comprend pas comme des technologies. La technologie descriptive, en revanche, est une discipline qu'il connaît parfaitement. "Les livres ne manquent pas ", dit-il, en citant le Dictionnaire technologique de Lenormand et Francœur, son Abrégé de Francœur, le Dictionnaire de l'industrie manufacturière d'Alexandre Baudrimont, le Portefeville industriel du Conservatoire de Claude Pouillet et César-Nicolas Leblanc, et, bien entendu, le Bulletin de la société d'encouragement. Mais, nous l'avons déjà mentionné, Lalanne a réduit cette vieille technographie a une série de faits divers. La vraie technologie se trouve dans «les livres qui traitent des applications des sciences à l'industrie » et il fait mention de l'économie industrielle de Babbage, de la technologie chimique de Jean-Baptiste Dumas (Chimie appliquée aux arts), et surtout de la pluralité des traités de la science des machines (Betancourt, Christian, Flachat, etc., voir note 47). À nouveau, il est clair que la technologie " à la Lalanne », c'est la science appliquée, surtout la mécanique appliquée.

\section{Épilogue. Laboulaye 1873. La consommation du tournant vers les sciences intermédiaires.}

En 1845, dans l'introduction à la première édition du Dictionnaire des arts et manufactures, arrangé d'après celui d'Andrew Ure ${ }^{49}$, Charles Laboulaye se montre disposé à définir la technologie comme science appliquée : "Qu'est-ce qu'un procédé industriel, sinon une application, une combinaison de lois physiques. Que peut-il donc y avoir de plus utile pour l'étude de la technologie ? Qu'y a-t-il de préférable pour rendre facile et complète la compréhension des procédés technologiques, que de les classer comme les applicatons des théories scientifiques? $11^{50}$

Seulement, il y a un problème. Beaucoup de sciences ne sont pas encore assez mûres pour être mises en relation fructueuse avec la pratique industrielle : "Malheureusement l'ensemble actuel des sciences et des arts n'est pas tellement complet, que la théorie existe toujours pour la pratique, sous forme scientifique $\|^{51}$.

Les sciences qui, selon Laboulaye, peuvent effectivement guider la pratique, sont la géométrie descriptive, la chimie, la métallurgie, la mécanique industrielle, la mécanique géométrique, disons la chimie et la sciences des machines. Laboulaye est tout près de Lalanne.

\section{Voir note 17.}

50 Charles Laboulaye, "Introduction ॥, Dictionnaire des arts et manufactures, Bruxelles, Decq, 1845, t. 1er, pp. i-viii. 51 lbid., p. vi. 
Mais en 1873, à l'occasion de la troisième édition de son Dictionnaire, Laboulaye prétend que les sciences ont suffisamment progressé pour servir de base à diverses technologies. Ce service passe par le truchement d'un type de sciences que Laboulaye appelle sciences de l'ingénieur ou sciences intermédiaires, expression réinventée par Hélène Vérin ${ }^{52}$ : « Les sciences étudiées uniquement au point de vue abstrait de l'explication des phénomènes, de la satisfaction intellectuelle qu'éprouve l'esprit quand il arrive à prévoir les phénomènes, leur ordre de succession, leur dépendance mutuelle, ne sont pas toujours essentiellement propres à être directement appliquées. Elles doivent souvent recevoir des développements spéciaux pour répondre aux divers cas de la pratique, être transformées en quelque sorte pour être mises sous une forme qui permette de les utiliser plus facilement. La nécessité de ce travail préparatoire est bien indiquée par la grande utilité des personnes qui sont chargées d'effectuer ce travail : nous voulons parler des ingénieurs. Leur tâche consiste à utiliser constamment les derniers progrès

52 Voir note 32. des sciences pour les traduire en progrès industriels et à transmettre aux fabricants, aux ouvriers, des projets dont l'exécution, des méthodes dont l'emploi rentrent dans les limites d'adresse ou d'expérience ordinaire. Cette classe de sciences intermédiaires, que l'on peut appeler sciences de l'ingénieur [...] $11^{53}$.

On peut distinguer cinq sciences intermédiaires ou technologies : physique industrielle, chimie industrielle, biologie industrielle, mécanique industrielle, géométrie industrielle. II n'est plus nécessaire de préférer la sciences des machines. La technologie chimique s'est pleinement développée et la biologie a détruit l'inexplicabilité de la force vitale, de sorte que les soins attentifs de l'agriculteur et de l'éleveur peuvent être rangés parmi les opérations techniques.

C'est ainsi que la définition de la technologie comme science intermédiaire, préparée par Lalanne dans les années 1840, est devenue courante dans les années 1870.

53 Charles Laboulaye, "Introduction », Dictionnaire des arts et manufactures, $3^{e}$ édition, Paris, 1873. 